\title{
Emergency response network design for hazardous materials transportation with uncertain demand
}

\author{
Elnaz Rezaei Ehsan*, Ahmad Makui, Kamran Shahanaghi
}

Department of Industrial Engineering, Iran University of Science \& Technology, Tehran, Iran

\begin{tabular}{|c|c|}
\hline A R T I C LE I NFO & ABSTRACT \\
\hline $\begin{array}{l}\text { Article history: } \\
\text { Received 15 January } 2012 \\
\text { Accepted April } 292012 \\
\text { Available online } \\
9 \text { May } 2012 \\
\text { Keywords: } \\
\text { Location-allocation } \\
\text { Multi objective model emergency } \\
\text { response } \\
\text { Hazardous materials } \\
\text { Uncertain demand }\end{array}$ & $\begin{array}{l}\text { Transportation of hazardous materials play an essential role on keeping a friendly environment. } \\
\text { Every day, a substantial amount of hazardous materials (hazmats), such as flammable liquids and } \\
\text { poisonous gases, need to be transferred prior to consumption or disposal. Such transportation may } \\
\text { result in unsuitable events for people and environment. Emergency response network is designed } \\
\text { for this reason where specialist responding teams resolve any issue as quickly as possible. This } \\
\text { study proposes a new multi-objective model to locate emergency response centers for } \\
\text { transporting the hazardous materials. Since many real-world applications are faced with } \\
\text { uncertainty in input parameters, the proposed model of this paper also assumes that reference and } \\
\text { demand to such centre is subject to uncertainty, where demand is fuzzy random. The resulted } \\
\text { problem formulation is modelled as nonlinear non-convex mixed integer programming and we } \\
\text { used NSGAII method to solve the resulted problem. The performance of the proposed model is } \\
\text { examined with several examples using various probability distribution and they are compared } \\
\text { with the performance of other existing method. }\end{array}$ \\
\hline
\end{tabular}

\section{Introduction}

During the past few decades, there have been significant amount of hazardous materials generated by human beings. Today, there is no doubt that some of damages caused by such materials are irreversible and we need to find good ways to reduce these damages as much as possible. According to US Department of Transportation (2000), a substantial amount of hazardous materials (hazmats), such as flammable liquids and poisonous gases, need to be transported prior to their consumption or disposal and a significant amount are transported using containers. For example, in the highways of North America, there are about 800000 road transportations for transporting the pollutants by containers per day. Therefore, there is an increasing demand for proposing suitable methods in this area.

Erkut and Gzara (2008) considered the problem of network design for hazardous material transportation where the government designates a network, and the carriers choose the routes on the network. They modeled the problem as a bi-level network flow formulation and analyzed the resulted problem in three different scenarios. Kara and Verter (2004) formulated the design problem as a bi-

* Corresponding author.

E-mail: rezaei.elnaz@hotmail.com (E. Rezaei)

(C) 2012 Growing Science Ltd. All rights reserved. doi: $10.5267 /$ j.ijiec.2012.05.001 
level mathematical programming model by assuming that the carriers would always use the shortest paths on the hazmat network. The objective for the authority was to choose the minimum risk network, taking into account the cost-minimizing behavior of the carriers. The model is relatively general since it does not limit the hazmat network to a tree. However, their resulting mathematical model is quite large and is not computationally feasible for large networks. Erkut and ALP (2007) designed a network as a tree, which creates an advantage when authorities decide to improve the roads' conditions in the network for hazardous materials transportation to reduce the risks and events such as repaving the roads and better lighting. However, it has a disadvantage because it is only applied for road transportation and in civil transportation; it may not be applicable due to incurring many costs; while this network design is relatively small and is optimal for networks with actual sizes.

Schütz et al. (2008) presented the problem of minimizing the expected cost of locating a number of single product facilities and allocating uncertain customer demand to this presented. The total costs consist of two components of linear transportation and the costs of investing in a facility as well as maintaining and operating it. These facility costs are general and non-linear in shape and could express both changing economies of scale and diseconomies of scale. They formulated the problem as a twostage stochastic programming model where both demand and short-run costs may be uncertain at the investment time. They used a solution method based on Lagrangean relaxation.

Yang et al. (2008) presented flow capturing location-allocation problem with stochastic demand under Hurwicz's rule where the assumption that the customer flow is stochastic variable in probability space, chance-constrained programming model is developed for this problem under Hurwicz's rule. To solve this stochastic model, stochastic simulation, greedy search and genetic algorithm were integrated to produce a hybrid intelligent algorithm. Ishii et al. (2007) proposed another model by considering the satisfaction degree with respect to the distance from the facility for each customer (residents) and preference of the site in an urban area.

The objective was to find the site of the facility, which maximizes the minimal satisfaction degree among all demand points and maximizes the preference of the site. Yang et al. (2007) investigated distribution centers location problem under fuzzy environment. Consequentially, chance-constrained programming model for the problem is designed and some properties of the model were investigated. Tabu search algorithm, genetic algorithm and fuzzy simulation algorithm were integrated to find the approximate best solution of the model. Wen and Iwamura (2008) proposed the FLA problem under random fuzzy environment using $(\alpha, \beta)$-cost minimization model under the Hurwicz's criterion. By varying the value $\lambda$, it can balance the optimistic level of the decision makers. For solving the random fuzzy model efficiently, the simplex algorithm, random fuzzy simulation and genetic algorithm were integrated to produce a hybrid intelligent algorithm. Wang and Watada (2010) studied a facility location model with fuzzy random parameters and its swarm intelligence approach. A value-at-risk (VaR) based fuzzy random facility location model (VaR-FRFLM) was built in which both the costs and demands were assumed to be fuzzy random variables, and the capacity of each facility was unfixed but a decision variable assuming continuous values. A hybrid modified particle swarm optimization (MPSO) approach was proposed to solve the VaR-FRFLM. A numerical experiment illustrated the application of the proposed hybrid MPSO algorithm and lays out its robustness to the system parameter settings. The comparison shows that the hybrid MPSO exhibits better performance than that when hybrid regular continuous-binary PSO and genetic algorithm (GA) are used to solve the VaR-FRFLM.

\section{The proposed study}

\subsection{General model of hybrid planning}

In the regular planning model, if one or several parameters have both probability properties and Fuzzy properties, the resulted model called "hybrid planning model", general hybrid planning model is as follows, 
$\max f(x, \varepsilon)$

subject to

$g_{i}(x, \varepsilon) \leq \circ, j=1,2, \ldots, n$

where, $\boldsymbol{x}$ is the decision vector, $\varepsilon$ is the hybrid vector, $f(x, \varepsilon)$ is the objective function and $g_{i}(x, \varepsilon)$ is the constraint functions for $j=1,2,3, \ldots ., n$.

\subsection{Expected value model}

The first type of hybrid planning model is the expected value model optimizing the expected objective function based on the expected constraints. For example, minimizing the expected cost, expected profit, etc., the expected value model is applied as follows

$\max E[f(x, \varepsilon)]$

subject to

$E\left[g_{i}(x, \varepsilon)\right] \leq 0, j=1, \ldots \ldots, n$

where $x$ is the decision vector, $\varepsilon$ is the hybrid vector, $f(x, \varepsilon)$ is the objective function and $g_{i}(x, \varepsilon)$ is the constraint functions for $j=1,2,3, \ldots . ., n$ and $E[]$ is the symbol of expected value.

There are literally different techniques to handle uncertainty associated with input data such as using fuzzy numbers but the fuzzy parameters themselves could have stochastic nature. Liu (2007) investigated project planning problem when there are Fuzzy-random variables during the time of implementing the activities and implemented hybrid intelligent algorithm for problem solving.

\subsection{Chance-Constrained Programming}

Planning with chance constrains as the second type of hybrid planning is a powerful tool for modeling the hybrid decision making systems assuming that hybrid limitations exist at least in $\gamma$ percentage of times, that $\gamma$ is a reliable level determining by decision maker. Planning model with chance constrains is as follows,

$\max \bar{f}$

subject to

$\operatorname{ch}\{f(x, \varepsilon) \geq \bar{f}\} \geq \gamma$

$\operatorname{ch}\left\{g_{i}(x, \varepsilon) \leq 0\right\} \geq \gamma, j=1, \ldots \ldots, n$

where $x$ is the decision vector, $\varepsilon$ is the combined vector, $f(x, \varepsilon)$ is the objective function and $g_{i}(x, \varepsilon)$ is the constraint functions for $j=1,2,3, \ldots, n$ and $\gamma$ is the reliability and $\operatorname{ch}\{\}$ is the chance measure. Wen \& Iwamura (2008) used this model to solve the location-allocation facility problem in a fuzzyrandom environment using a hybrid intelligent algorithm. Liu (2007) studied the project planning issue when times of implementing the activities are fuzzy-random variables with an adaptation of hybrid intelligent algorithm.

\subsection{Dependent-Chance Programming}

Practically, there are multiple tasks in a typical complicated combined decision system and occasionally decision maker requires maximizing the chances of such tasks. The proposed fuzzy random method proposed by Liu (2007) has the following form, 
$\max \operatorname{ch}\{f(x, \varepsilon) \geq \bar{f}\}$

subject to

$\left\{g_{i}(x, \varepsilon) \leq 0\right\} \geq \gamma, j=1, \ldots \ldots, n$

where $x$ is the decision vector, $\varepsilon$ is the combined vector, $f(x, \varepsilon)$ is the goal function and $g_{i}(x, \varepsilon)$ is the constraint functions for $j=1,2,3, \ldots ., n$ and $\gamma$ is the reliability and $\operatorname{ch}\{\}$ is the chance measure. There are literally different applications for the implementation of fuzzy random technique. Liang et al. (2007) considered chance-related two-level planning model and used fuzzy random to detect the sources using combined intelligent algorithm as solution procedure. Liu (2007) studied the project planning issue where the activities' times were fuzzy-random variables. Sadjadi et al. (2012) investigated the project critical path problem in an environment with hybrid uncertainty, where the duration of activities were treated with random fuzzy variables. They implemented chance constraints programming to obtain a robust critical path and converted the resulted problem into a deterministic model in two stages. In the first stage, the uncertain model was converted into a model with interval parameters by alpha-cut method and distribution function concepts and the interval model was converted to a deterministic model by robust optimization and min-max regret criterion. Since the resulted problem formulation was NP-Hard, a genetic algorithm with a proposed exact algorithm were used to solve the final model.

\section{The proposed method}

The proposed model of this paper uses fuzzy-random to handle uncertainty associated with demand for locating the emergency facilities for transporting the hazardous materials with three objective functions. Assumptions, parameters and decision variables used in this model includes as follows, Model Assumptions:

- Emergency response centers have been well equipped;

- Emergency response centers and demand point in the network are accessible;

- Any node indicates an emergency response center or a demand point;

- Facilities only can be established on the nodes;

- Any demand point only can give service from a facility but any facility may take service to several demand points.

\subsection{Sets, Parameters and Variables of Problem}

$\mathrm{N}(\mathrm{V}, \mathrm{A})$ : Emergency response network

$\mathrm{V}$ : nodes set

A: Arcs set

$\mathrm{V}=\mathrm{EUO}$

$\mathrm{E}(1,2, \ldots \mathrm{e})$ : is potential emergency response center node;

$\mathrm{O}(1,2, \ldots \mathrm{O})$ : is transport nodes

P: No. of facilities that must be located;

$a_{i}$ : is the fixed cost of establish emergency response center at node $i$

$b_{i}$ : is the unit cost of transportation of emergency response center $i$

$\mathrm{V}_{\mathrm{i}}$ : is the average vehicle speed of emergency response center $\mathrm{i}$

$\mathrm{e}_{\mathrm{ij}}$ : is the distance from emergency response center I to node $\mathrm{j}, i \in E$ and $j \in O$

$w_{j}$ : demand weight of node $\mathbf{j}$

$\widehat{r}_{j}$ : small radius defined for distance of demand node $\mathrm{j}$ from nearest response center;

$\tilde{r}_{j}$ : great radius defined for distance of demand node $\mathrm{j}$ from nearest response center; 
$f_{j}\left(e_{i j}\right)$ : covering area of demand node $\mathrm{j}$ as a fractional function

$g_{i}(i)$ :a part of demand weight of node $\mathrm{j}$ made by facility and provided in node $\mathrm{i}$

Note that Berman et al. (2011) stated that for any demand node, there are defined two coverage radius; the demand originating from a node is considered fully covered; if the shortest distance between demand point to facility from smaller radius is not greater, and not covered at all, if the shortest distance between demand point to facility of greater radius is not shorter. For distance between these two radiuses, the coverage level is defined as fractional function $f_{j}\left(e_{i j}\right)$, therefore, $g_{j}(i)$ is calculated as follows,

$g_{j}(i):\left\{\begin{array}{cc}1 & e_{i j} \leq \widehat{r}_{j} \\ f_{j}\left(e_{i j}\right) & \widehat{r}_{j} \leq e_{i j} \leq \tilde{r}_{j} \\ 0 & e_{i j} \geq \tilde{r}_{j}\end{array}\right.$

and $f_{j}\left(e_{i j}\right)$ is calculated by Thales theorem as follows,

$f_{j}\left(e_{i j}\right)=\frac{\tilde{r}_{j}-e_{i j}}{\tilde{r}_{j}-\hat{r}_{i}}$,

where:

$x_{i}=\left\{\begin{array}{lc}1 & \text { if emergency response is established } \\ 0 & \text { otherwise }\end{array} y_{i j}= \begin{cases}1 & \text { if facility } i \text { is assigned to demand point } j \\ 0 & \text { otherwise }\end{cases}\right.$

The mathematical planning model of emergency response centers location-allocation with 3 objective functions is as follows,

$\min \sum_{i \in E} e_{i j} \frac{x_{i}}{v_{i}}$

$\min \sum_{i \in E} a_{i} x_{i}+\sum_{i \in E} \sum_{j \in O} b_{i} e_{i j} y_{i j}$

$\max \sum_{i \in E} \sum_{j \in O} g_{j}(i) w_{j} v_{i} \frac{y_{i j}}{e_{i j}}$

subject to

$\sum_{i \in E} y_{i j}=1 \quad, \forall j \quad y_{i j} \leq x_{i} \quad, \forall i, j \quad \sum_{i \in E} x_{i}=P$

$x_{i} \in\{o, 1\}, y_{i j} \in\{o, 1\}$

The first objective function of problem is associated with time, which minimizes the total time of transportation; the second one minimizes the total cost of transportation and the fixed cost of opening centers and the third objective function maximizes the environmental safety. The first constraint states that any demand point can only give service from a facility; the second constraint states that allocating the demand point to another point is possible only when a facility point's already been established. The third constraint states the number of facilities that must be established and fourth constraint indicates the type of decision variables. 
According to explanations provided before, hybrid Chance-Constrained Programming for this model as below:

$\min \sum_{i \in E} e_{i j} \frac{x_{i}}{v_{i}}$

$\min \sum_{i \in E} a_{i} x_{i}+\sum_{i \in E} \sum_{j \in O} b_{i} e_{i j} y_{i j}$

$\max \bar{f}$

subject to

$\operatorname{ch}\left\{\sum_{i \in E} \sum_{j \in O} g_{j}(i) w_{j} v_{i} \frac{y_{i j}}{e_{i j}}\right\} \geq \gamma$

$\sum_{i \in E} y_{i j}=1 \quad, \forall j \quad y_{i j} \leq x_{i} \quad, \forall i, j \quad \sum_{i \in E} x_{i}=P$

$x_{i} \in\{o, 1\}, y_{i j} \in\{o, 1\}$

where $\gamma$ is the reliability level for providing the provision $\left\{\sum_{i \in E} \sum_{j \in O} g_{i}(i) w_{j} v_{i} y_{i j} / e_{i j} \geq \bar{f}\right\}$ made by decision maker and the third objective function is the maximization of $\bar{f}$. The hybrid Dependent-Chance Programming of this problem is as follows,

$\min \sum_{i \in E} e_{i j} \frac{x_{i}}{v_{i}}$

$\min \sum_{i \in E} a_{i} x_{i}+\sum_{i \in E} \sum_{j \in O} b_{i} e_{i j} y_{i j}$

$\max c h\left\{\sum_{i \in E} \sum_{j \in O} g_{j}(i) w_{j} v_{i} \frac{y_{i j}}{e_{i j}} \geq \bar{f}\right\}$

subject to

$\sum_{i \in E} y_{i j}=1 \quad, \forall j \quad y_{i j} \leq x_{i} \quad, \forall i, j \quad \sum_{i \in E} x_{i}=P$

$x_{i} \in\{o, 1\}, y_{i j} \in\{o, 1\}$

where $\bar{f}$ is an area of objective function obtained by decision maker and the third objective function indicates that the environmental safety may be increased according to the coverage of demand points and speed and distance between points from given value $\bar{f}$. When there is not enough knowledge about decision maker from the environmental conditions in models (8) and (9) we may have some unacceptable solution for decision maker or not optimal solution by choosing inappropriate value for $\bar{f}$ and $\gamma$.

For this reason, by providing a new model in a hybrid space, where $\gamma$ and $\bar{f}$ are optimized simultaneously, we may relieve the resulted complications. In the hybrid model, the maximization of $\bar{f}$ is considered in the first level from the third objective function and the maximization of $\gamma$ is considered in the second level of third objective function. In order for combining two models, one may use the same constraints. For this reason, in the Dependent-Chance Programming model, we enter the constraints as follows, 
$\min \sum_{i \in E} e_{i j} \frac{x_{i}}{v_{i}}$

$\min \sum_{i \in E} a_{i} x_{i}+\sum_{i \in E} \sum_{j \in O} b_{i} e_{i j} y_{i j}$

$\max \gamma$

subject to

$\operatorname{ch}\left\{\sum_{i \in E} \sum_{j \in O} g_{j}(i)_{w_{j}} v_{i} \frac{y_{i j}}{e_{i j}} \geq \bar{f}\right\} \geq \gamma \quad \sum_{i \in E} y_{i j}=1 \quad, \forall j \quad y_{i j} \leq x_{i} \quad, \forall i, j \quad \sum_{i \in E} x_{i}=P$

$x_{i} \in\{o, 1\}, y_{i j} \in\{o, 1\}$

Now we combine two models. We put the maximization $\bar{f}$ in the first level and maximization $\gamma$ in the second level from the third objective function, because this function aims to find an answer, which maximizes $\bar{f}$ such that maximizing the possibility of attaining to this value.

$\min \sum_{i \in E} e_{i j} \frac{x_{i}}{v_{i}}$

$\min \sum_{i \in E} a_{i} x_{i}+\sum_{i \in E} \sum_{j \in O} b_{i} e_{i j} y_{i j}$

$\max \bar{f}$

$\max \gamma$

subject to

$\operatorname{ch}\left\{\sum_{i \in E} \sum_{j \in O} g_{i}(i) w_{j} v_{i} y_{i j} / e_{i j} \geq \bar{f}\right\} \geq \gamma$

$\sum_{i \in E} y_{i j}=1, x_{i} \geq y_{i j}, \sum_{i \in E} x_{i}=P, x_{i} \in\{0,1\}, y_{i j} \in(0,1\} \quad \forall_{j}$

A method provided for solving the model is to combine two levels using the linear combination of two levels as an objective function. Now, in order to combine two levels, we set the first and the second goal functions a side temporarily and conduct the following operation:

Consider model (11) by considering $f_{\gamma}=\max \bar{f}$ as the objective function. If $\gamma_{1} \leq \gamma_{2}$, in this case, the solution space in $\gamma=\gamma_{1}$ is greater than the solution space in $\gamma=\gamma_{2}$. Therefore, $f_{\gamma_{1}}$ is not worse than $f_{\gamma_{2}}$, so $f_{\gamma_{1}} \geq f_{\gamma_{2}}$. Now, according to $\gamma \leq 1$, we consider $f_{\gamma} \geq f_{1}$. Thus, if in the problem, instead of studying different $\bar{f}$, we only study $\bar{f}$, which is greater than $\mathrm{f}_{1}$ (limiting the set of $\bar{f}$ ), it may not change the solution, because optimal value of $\bar{f}$, i.e. $\mathrm{f}_{\gamma}$ is greater than $\mathrm{f}_{1}$. Decision maker is also asked to determine a level of $\gamma$ that may not become worse. This value $\gamma$ is called $\gamma_{\mathrm{w}}$ and its correspondent is called $\mathrm{f}_{\mathrm{w}}$. We may use again from limiting the set of $\bar{f}$, and study ones that are less than $\mathrm{f}_{\mathrm{w}}$. Therefore, we use following,

$\mathrm{f}_{1} \leq \overline{\mathrm{f}} \leq \mathrm{f}_{\mathrm{w}}$

Now, because $-f_{1}+\bar{f} \geq 0$ and $-f_{1}+\bar{f} \leq-f_{1}+f_{w}$, we have,

$0 \leq \bar{f}-f_{1} \leq f_{w}-f_{1} \underset{f_{w}-f_{1} \geq 0}{\longrightarrow} 0 \leq \frac{\bar{f}-f_{1}}{f_{w}-f_{1}} \leq 1$

Because, this problem aims to increase the $\bar{f}$, therefore, $\frac{\bar{f}-f_{1}}{f_{w}-f_{1}}$ is also increased. Then, the third objective function of the problem is given by combining two levels: 
$\max \left\{\frac{\bar{f}-f_{1}}{f_{w}-f_{1}}\right\} \& 0 \leq \frac{\bar{f}-f_{1}}{f_{w}-f_{1}} \leq 1, \max \{\gamma\} \& 0 \leq \gamma \leq 1 \stackrel{0 \leq \lambda \leq 1}{\longrightarrow} \max \lambda \frac{\bar{f}-f_{1}}{f_{w}-f_{1}}+(1-\lambda) \gamma$

In this case, model is obtained in terms of Eq. (15).

$\min \sum_{i \in E} e_{i j} \frac{x_{i}}{v_{i}}$

$\min \sum_{i \in E} a_{i} x_{i}+\sum_{i \in E} \sum_{j \in O} b_{i} e_{i j} y_{i j}$

$\max \lambda \frac{\bar{f}-f_{1}}{f_{w}-f_{1}}+(1-\lambda) \gamma$

subject to

$\operatorname{ch}\left\{\sum_{i \in E} \sum_{j \in O} g_{j}(i) w_{j} v_{i} y_{i j} / e_{i j} \geq \bar{f}\right\} \geq \gamma$

$\sum_{i \in E} y_{i j}=1 \quad \forall_{j} \quad x_{i} \geq y_{i j} \quad \sum_{i \in E} x_{i}=P \quad \gamma \geq \gamma_{w}$

$x_{i} \in\{0,1\}, y_{i j} \in(0,1\}$

where, in this model, besides parameters as explained before, $\gamma$ is the reliability, $\gamma_{\mathrm{w}}$ is the minimum reliability level acceptable for decision maker, $\mathrm{f}_{\mathrm{w}}$ is maximum value of $\bar{f}$. Based on $\gamma=\gamma_{w}$, obtained by solving model (3-9), and $f_{1}$ is minimum value of $\bar{f}$ based on $\alpha=1$ obtaining by solving model (3-9). In this model, we give suitable values to $\lambda$; and because $\gamma$ must be less than the weight $\frac{\bar{f}-f_{1}}{f_{w}-f_{1}}$, these values must be selected between 0.5 and 1 . In this model, the weight of demand of points $w_{j}$ has been considered based on any $j$ of hybrid variable. For arranged m-array of $\left(\theta_{1}, \ldots \ldots . ., \theta_{m}\right) \in\left(\Theta_{1}, \ldots \ldots . ., \Theta_{w}\right)$, membership function is defined as $\mu\left(\theta_{1}, \ldots \ldots \ldots \theta_{m}\right)=\min \mu_{i}\left(\theta_{i}\right)$. According to Liu (2007), the chance for having the provision $\left\{\sum_{i \in E} \sum_{j \in O} g_{j}(i) w_{j} v_{i} y_{i j} / e_{i j} \geq \bar{f}\right\}$ is as follows,

$\operatorname{Ch}\{\Lambda\}=\left\{\begin{aligned} \sup _{\theta \in \Theta}\left(\frac{\min \mu_{i}\left(\theta_{i}\right)}{2} \wedge \operatorname{Pr}\left\{\sum_{i \in E} \sum_{j \in O} g_{j}(i) w_{j}(\theta) v_{i} y_{i j} / e_{i j} \geq \bar{f}\right\}\right) \\ \text { if } \sup _{\theta \in \Theta}\left(\frac{\min \mu_{i}\left(\theta_{i}\right)}{2} \wedge \operatorname{Pr}\left\{\sum_{i \in E} \sum_{j \in O} g_{j}(i) w_{j}(\theta) v_{i} y_{i j} / e_{i j} \geq \bar{f}\right\}\right)<0.5 \\ 1-\sup _{\theta \in \Theta}\left(\frac{\min \mu_{i}\left(\theta_{i}\right)}{2} \wedge \operatorname{Pr}\left\{\sum_{i \in E} \sum_{j \in O} g_{j}(i) w_{j}(\theta) v_{i} y_{i j} / e_{i j} \leq \bar{f}\right\}\right) \\ \text { if } \sup _{\theta \in \Theta}\left(\frac{\min \mu_{i}\left(\theta_{i}\right)}{2} \wedge \operatorname{Pr}\left\{\sum_{i \in E} \sum_{j \in O} g_{j}(i) w_{j}(\theta) v_{i} y_{i j} / e_{i j} \geq \bar{f}\right\}\right) \geq 0.5\end{aligned}\right.$

The proposed model of this paper can be solved using a typical genetic algorithm NSGAII and details of the computations are given in (Javanshir et al., 2012; Venkata Rao \& Patel, 2012).

\section{Case study and data analysis}

In this part, the actual data of transporting the hazardous materials in Isfahan province has been used and is solved by the proposed model. It must be mentioned that demand for different points is considered as random fuzzy and the distribution of the parameters are considered stochastic. In this case study, there are 6 emergency response nodes where 4 nodes must be selected 4 and facilities are located in these places. In addition, there are 10 demand nodes, which are candidates for transportation accident. These points may include origin nodes or destination nodes or nodes in the transportation route. Fig 1 shows details of our proposed case study._In this network, star shapes are demand nodes 
and circular shapes are response emergency potential nodes and the nodes of 1,4 and 10 are positions of refineries. The name of cities is shown in the Table 1 as follows.

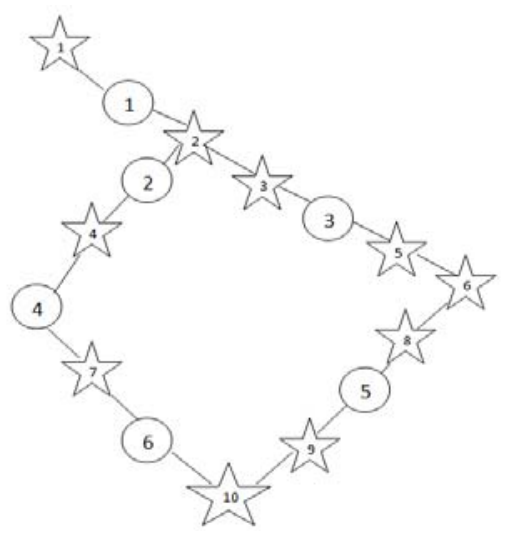

Fig. 1. The network design for the Isfahan province

Table 1

The City of network

\begin{tabular}{|c|c|c|c|c|c|c|c|c|c|c|}
\hline Node & 1 & 2 & 3 & 4 & 5 & 6 & 7 & 8 & 9 & 10 \\
\hline city & $\begin{array}{l}5 \mathrm{Km} \text { of } \\
\text { Tehran } \\
\text { road }\end{array}$ & Kashan & Ghasemabad & Meimeh & Sahamiye & Ardesten & Razaviye & Balgham & Habibabad & Isfahan \\
\hline
\end{tabular}

Table 2 and Table 3 show the information associated with emergency response and demand notes, respectively.

Table 2

The data of emergency response nodes

\begin{tabular}{lllllll}
\hline Node & 1 & 2 & 3 & 4 & 5 & 6 \\
\hline $\mathrm{a}_{\mathrm{i}}$ & 25 & 20 & 15 & 25 & 20 & 20 \\
$\mathrm{~b}_{\mathrm{i}}$ & 30 & 25 & 20 & 25 & 20 & 20 \\
$\mathrm{v}_{\mathrm{i}}$ & 60 & 60 & 60 & 60 & 60 & 60 \\
\hline
\end{tabular}

Table 3

The data of demand nodes

\begin{tabular}{lllllllllll}
\hline Node & 1 & 2 & 3 & 4 & 5 & 6 & 7 & 8 & 9 & 10 \\
\hline$\widehat{r}_{j}$ & 35 & 35 & 35 & 40 & 30 & 40 & 25 & 30 & 30 & 30 \\
$\tilde{r}_{j}$ & 45 & 45 & 40 & 45 & 40 & 45 & 45 & 40 & 45 & 45 \\
\hline
\end{tabular}

Distance between demand nodes and candidate nodes for establishing the emergency facilities has been given in Table 4, which were computed based on the distance of towns of Isfahan province.

\section{Table 4}

Distance between nodes

\begin{tabular}{lllllllllll}
\hline $\mathrm{i}$ & & & & & $\mathrm{j}$ & & & \\
\hline 1 & 30 & 35 & 62 & 100 & 112 & 136 & 160 & 166 & 218 \\
2 & 100 & 35 & 62 & 30 & 112 & 137 & 90 & 166 & 148 & 120 \\
3 & 117 & 52 & 25 & 117 & 25 & 50 & 177 & 79 & 149 & 177 \\
4 & 160 & 95 & 122 & 30 & 172 & 187 & 30 & 158 & 88 & 60 \\
5 & 231 & 166 & 139 & 152 & 89 & 64 & 92 & 35 & 35 & 62 \\
6 & 205 & 140 & 167 & 75 & 167 & 142 & 15 & 113 & 43 & 15 \\
\hline
\end{tabular}

We have also assumed the following, 
$g_{1}(1)=g_{2}(1)=g_{2}(2)=g_{4}(2)=g_{3}(3)=g_{5}(3)=g_{4}(4)=g_{7}(4)=g_{8}(5)=g_{9}(5)=g_{7}(6)=g_{10}(6)=1$, $\boldsymbol{g}_{3}(1)=\boldsymbol{g}_{3}(2)=\frac{3}{35}, g_{2}(3)=\frac{12}{25}, \boldsymbol{g}_{6}(3)=\frac{1}{2}, \boldsymbol{g}_{10}(5)=\frac{2}{35}, \boldsymbol{g}_{9}(6)=\frac{11}{15}$ and all other $\boldsymbol{g}_{j}(i)$ are equal to zero. In this example, it is assumed that the weight of any demand node follows of Poison distribution and the mean of these weights that are mean of Poison mean is Fuzzy. We consider the weight of demand nodes as well as means according to following table and as Fuzzy triangle numbers.

Table 5

The weight of nodes with poison distribution $P(\lambda)$

\begin{tabular}{llllll}
\hline Node & 1 & 2 & 3 & 4 & 5 \\
\hline$w_{j}$ & $\lambda=(10,11,12)$ & $\lambda=(13,14,15)$ & $\lambda=(14,15,16)$ & $\lambda=(17,18,19)$ & $\lambda=(16,17,18)$ \\
Node & 6 & 7 & 8 & 9 & 10 \\
$w_{j}$ & $\lambda=(11,12,13)$ & $\lambda=(13,14,15)$ & $\lambda=(17,18,19)$ & $\lambda=(17,18,19)$ & $\lambda=(13,14,15)$ \\
\hline
\end{tabular}

where $\mathrm{p}(\lambda)$ represents the Poison Distribution with mean $\lambda$ and $(a, b, c)$ indicate the triangular fuzzy number. Also we have assumed that decision maker wants an answer with the chance less than 0.6, i.e. $\gamma_{\mathrm{w}}=0.6$. Using model (12), we can calculate the values $\mathrm{f}_{1}$ and $\mathrm{f}_{0.6}$ for $\gamma=1$ and $\gamma=0.6$ respectively, which yields $f_{1}=1, f_{0.6}=128$. Now, the third goal function of model (16) is as follows,

$\max \lambda \frac{\bar{f}-1}{128-1}+(1-\lambda) \gamma$

We assign different values to $\lambda$, and calculate the values of variables and objective functions. Initially, we assign 0.7 to $\lambda$. In this case, we find 5 solutions, which are summarized in Table 6 and Table 7 shows the locations.

\section{Table 6}

Different values of objective functions for $\lambda=0.7$

\begin{tabular}{lllccc}
\hline Solution & $\bar{f}$ & $\gamma$ & First objective function & Second objective function & Third objective function \\
\hline 1 & 221 & 0.77 & 45.9444 & 14520 & 0.7830 \\
2 & 301 & 0.74 & 45.7222 & 14705 & 0.9747 \\
3 & 318 & 0.73 & 46.1333 & 12690 & 1.0129 \\
4 & 323 & 0.76 & 45.6654 & 13000 & 1.0143 \\
5 & 220 & 0.75 & 46.9444 & 12860 & 0.7745 \\
\hline
\end{tabular}

Table 7

Selected nodes for $\lambda=0.7$

\begin{tabular}{lllllllllll}
\hline & $\boldsymbol{X}_{1}$ & $\boldsymbol{X}_{2}$ & $\boldsymbol{X}_{3}$ & $\boldsymbol{X}_{4}$ & $\boldsymbol{X}_{5}$ & $\boldsymbol{X}_{6}$ & $\boldsymbol{X}_{7}$ & $\boldsymbol{X}_{8}$ & $\boldsymbol{X}_{9}$ & $\boldsymbol{X}_{10}$ \\
\hline Solution1 & 0 & 1 & 1 & 1 & 1 & 0 & 0 & 0 & 0 & 0 \\
Solution2 & 0 & 1 & 1 & 0 & 1 & 1 & 0 & 0 & 0 & 0 \\
Solution3 & 0 & 1 & 1 & 1 & 0 & 1 & 0 & 0 & 0 & 0 \\
Solution4 & 0 & 1 & 1 & 1 & 0 & 1 & 0 & 0 & 0 & 0 \\
Solution5 & 0 & 1 & 1 & 1 & 0 & 1 & 0 & 0 & 0 & 0 \\
\hline
\end{tabular}

Now, suppose $\lambda=0.8$, in this case, there are three obtained solutions and the results are summarized in Table 8 and Table 9.

\section{Table 8}

Different values of objective functions for $\lambda=0.8$

\begin{tabular}{llrccc}
\hline Solution No. & $\bar{f}$ & $\gamma$ & First objective function & Second objective function & Third objective function \\
\hline 1 & 311 & 0.7 & 45.0556 & 12135 & 1.0409 \\
2 & 323 & 0.72 & 45.7222 & 14725 & 1.0673 \\
3 & 320 & 0.74 & 45.7222 & 11865 & 1.0111 \\
\hline
\end{tabular}


Table 9

Selected nodes for $\lambda=0.8$

\begin{tabular}{lllllllllll} 
& $\boldsymbol{X}_{1}$ & $\boldsymbol{X}_{2}$ & $\boldsymbol{X}_{3}$ & $\boldsymbol{X}_{4}$ & $\boldsymbol{X}_{5}$ & $\boldsymbol{X}_{6}$ & $\boldsymbol{X}_{7}$ & $\boldsymbol{X}_{8}$ & $\boldsymbol{X}_{9}$ & $\boldsymbol{X}_{10}$ \\
\hline Solution1 & 1 & 0 & 1 & 0 & 1 & 1 & 0 & 0 & 0 & 0 \\
Solution2 & 0 & 1 & 1 & 0 & 1 & 1 & 0 & 0 & 0 & 0 \\
Solution3 & 0 & 1 & 1 & 0 & 1 & 1 & 0 & 0 & 0 & 0 \\
\hline
\end{tabular}

If we would like to compare three sets of obtained solutions for different $\lambda$, we can use the diversity and spacing metrics. These metrics are shown in the table 10 for any given set of solutions.

\section{Table10}

Comparison of the diversity and spacing metrics for the three solution sets

\begin{tabular}{lll}
\hline & diversity & spacing \\
\hline The first set & 97.0052 & 0.1463 \\
The second set & 91.1592 & 1.4824 \\
\hline
\end{tabular}

We can find out that the first set is better because it represents more diversity metric and less spacing. Now we can decide which nodes can be selected by considering which optimal objective function is more important for decision maker. If we need to select one of the solutions, we may choose the forth one because it has the best parameter in both objective functions. Therefore, the facilities have been located in nodes 2, 3, 4 and 6. In order to better analyze the behavior of the proposed model we consider another scenario. This time it is assumed that the weight of any demand node follows from uniform distribution as indicated in following Table 11. In this table $U(a, b)$ indicates the Uniform distribution and $(a, b, c)$ indicates triangular Fuzzy numbers. Assume also that this time the decision maker wants an answer with minimum chance from 0.7 , i.e. $\gamma_{\mathrm{w}}=0.7$. Using model (12), we can calculate $\mathrm{f}_{1}$ and $\mathrm{f}_{0.7}$ values and its results are as $f_{1}=3$ and $f_{0.7}=45$. Now, the third goal function of model (16) is as $\max \lambda \frac{\bar{f}-3}{45-3}+(1-\lambda) \gamma$.

Table 11

The weight of nodes

\begin{tabular}{lccccc}
\hline Node & 1 & 2 & 3 & 4 & 5 \\
\hline$W_{j}$ & $U\left(1+x_{1}, 6+x_{1}\right)$ & $U\left(2+x_{1}, 5+x_{1}\right)$ & $U\left(1+x_{1}, 5+x_{1}\right)$ & $U\left(2+x_{1}, 5+x_{1}\right)$ & $U\left(3+x_{1}, 5+x_{1}\right)$ \\
& $x_{1}=(5,6,7)$ & $x_{1}=(4,5,6)$ & $x_{1}=(6,7,8)$ & $x_{1}=(3,4,5)$ & $x_{1}=(3,4,5)$ \\
\hline Node & 6 & 7 & 8 & 9 & 10 \\
$W_{j}$ & $U\left(1+x_{1}, 5+x_{1}\right)$ & $U\left(2+x_{1}, 8+x_{1}\right)$ & $U\left(1+x_{1}, 8+x_{1}\right)$ & $U\left(2+x_{1}, 6+x_{1}\right)$ & $U\left(2+x_{1}, 5+x_{1}\right)$ \\
& $x_{1}=(4,5,6)$ & $x_{1}=(5,6,7)$ & $x_{1}=(3,4,5)$ & $x_{1}=(4,5,6)$ & $x_{1}=(3,4,5)$ \\
\hline
\end{tabular}

Therefore, we assign 0.7 to $\lambda$ and calculate the values of variables and objective functions. In this case, there have obtained 4 Solution s indicated in following table.

Table 12

values of objective functions for $\lambda=0.7$

\begin{tabular}{lllccc}
\hline Solution No. & $\bar{f}$ & $\gamma$ & First objective function & Second objective function & Third objective function \\
\hline 1 & 167 & 0.77 & 68.5833 & 8855 & 2.8719 \\
2 & 171 & 0.8 & 72.0833 & 9670 & 2.9445 \\
3 & 168 & 0.72 & 70.2833 & 8860 & 2.8728 \\
4 & 170 & 0.77 & 72.0833 & 8920 & 2.9196 \\
\hline
\end{tabular}


Note that when $\lambda$ is given by a bigger value, the problem in the third objective function tries to reduce the value $\gamma$ and increases $\bar{f}$ and because decision maker asks for probability value more than 0.7 , if we obtain some solutions, and the obtained value $\gamma$ is less than this value, we might ignore it. According to obtained parameters for the objective functions, whether the first optimal objective function or the second one is desirable, solution 1 is selected and if the third one is desirable, we should select solution 2. It depends on decision maker and we can choose the first answer because it improves two objective functions and decide to locate the facilities in parts 2, 3, 5 and 6.

Table 13

Selected nodes for $\lambda=0.7$

\begin{tabular}{lllllllllll}
\hline & $\boldsymbol{X}_{1}$ & $\boldsymbol{X}_{2}$ & $\boldsymbol{X}_{3}$ & $\boldsymbol{X}_{4}$ & $\boldsymbol{X}_{5}$ & $\boldsymbol{X}_{6}$ & $\boldsymbol{X}_{7}$ & $\boldsymbol{X}_{8}$ & $\boldsymbol{X}_{9}$ & $\boldsymbol{X}_{10}$ \\
\hline Solution1 & 0 & 1 & 1 & 0 & 1 & 1 & 0 & 0 & 0 & 0 \\
Solution2 & 1 & 0 & 1 & 0 & 1 & 1 & 0 & 0 & 0 & 0 \\
Solution3 & 0 & 0 & 1 & 1 & 1 & 1 & 0 & 0 & 0 & 0 \\
Solution4 & 1 & 0 & 1 & 0 & 1 & 1 & 0 & 0 & 0 & 0 \\
\hline
\end{tabular}

Now we use the data from the literature (Wang \& Vatada, 2010) and solve the proposed model once more. In this case, there are 5 demands and 10 candidate points of establishment and four facilities are located in establishment nominated points. Table 14 shows the distance from point $i$ to demand point $j$.

\section{Table 14}

Distance from point $i$ to demand point $j$

\begin{tabular}{ccccccccccc}
\hline & \multicolumn{10}{c}{$\mathrm{i}$} \\
$\mathrm{j}$ & 1 & 2 & 3 & 4 & 5 & 6 & 7 & 8 & 9 & 10 \\
\hline 1 & 16 & 21 & 19 & 18 & 14 & 18 & 16 & 20 & 18 & 20 \\
2 & 17 & 15 & 17 & 14 & 18 & 16 & 17 & 18 & 17 & 14 \\
3 & 24 & 20 & 25 & 22 & 23 & 22 & 24 & 22 & 20 & 22 \\
4 & 19 & 22 & 18 & 15 & 21 & 17 & 22 & 21 & 22 & 16 \\
5 & 13 & 10 & 16 & 13 & 14 & 11 & 13 & 15 & 14 & 13 \\
\hline
\end{tabular}

The weight amount of fuzzy-random demand points are given in the Table 15.

Table 15

The weight of nodes

\begin{tabular}{lccccc}
\hline Node & 1 & 2 & 3 & 4 & 5 \\
\hline (a,b,c) & $\left(20+x_{1}, 22+x_{1}, 23+x_{1}\right)$ & $\left(18+x_{1}, 20+x_{1}, 21+x_{1}\right)$ & $\left(16+x_{1}, 18+x_{1}, 19+x_{1}\right)$ & $\left(22+x_{1}, 23+x_{1}, 24+x_{1}\right)$ & $\left(20+x_{1}, 22+x_{1}, 23+x_{1}\right)$ \\
Unif. Dist. & $x_{1} \sim U(1,2)$ & $x_{2} \sim U(1,3)$ & $x_{3} \sim U(2,4)$ & $x_{4} \sim U(2,3)$ & $x_{5} \sim U(3,4)$ \\
& & & &
\end{tabular}

In Table 15, $(\mathrm{a}, \mathrm{b}, \mathrm{c})$ indicates triangular fuzzy numbers and $U(a, b)$ indicates the Uniform distribution. Assume also that this time the decision maker wants an answer with minimum chance of 0.7 , i.e. $\gamma_{\mathrm{w}}=$ 0.7. The implementation of the proposed model yields $f_{1}=1$ and $f_{0.7}=339$. Now, third goal function of model (3-12) is as below:

$\max \lambda \frac{\bar{f}-1}{339-1}+(1-\lambda) \gamma$

Therefore, we assign 0.7 to $\lambda$ and calculate the values of variables and objective functions. In this case, there have obtained 5 Solution s indicated in Table 16 and Table 17 as follows, 
Table 16

The values of objective functions

\begin{tabular}{lllccc}
\hline Solution No. & $\bar{f}$ & $\gamma$ & First objective function & Second objective function & Third objective function \\
\hline 1 & 96 & 0.98 & 5.6833 & 2192 & 0.4714 \\
2 & 325 & 0.78 & 5.8500 & 2001 & 0.8197 \\
3 & 324 & 0.82 & 5.8000 & 2247 & 0.8041 \\
4 & 315 & 0.77 & 6.0667 & 2181 & 0.6194 \\
5 & 100 & 0.86 & 5.7833 & 2176 & 0.43 \\
\hline
\end{tabular}

Table 17

The selected nodes

\begin{tabular}{|c|c|c|c|c|c|c|c|c|c|c|}
\hline & $X_{1}$ & $X_{2}$ & $X_{3}$ & $X_{4}$ & $X_{5}$ & $X_{6}$ & $X_{7}$ & $X_{8}$ & $X_{9}$ & $X_{10}$ \\
\hline Solution1 & & & & $\sqrt{ }$ & $\sqrt{ }$ & $\sqrt{ }$ & & & $\sqrt{ }$ & \\
\hline Solution2 & & & & $\sqrt{ }$ & $\sqrt{ }$ & $\sqrt{ }$ & $\sqrt{ }$ & & & \\
\hline Solution3 & & & & $\sqrt{ }$ & $\sqrt{ }$ & $\sqrt{ }$ & $\sqrt{ }$ & & & \\
\hline Solution4 & & $\sqrt{ }$ & & $\sqrt{ }$ & $\sqrt{ }$ & $\sqrt{ }$ & & & & \\
\hline Solution5 & & & & $\sqrt{ }$ & $\sqrt{ }$ & $\sqrt{ }$ & & & & $\sqrt{ }$ \\
\hline
\end{tabular}

Note that Wang and Watada (2010) reported that the facility should be located in the 2, 4, 5 and 9 candidate points with the objective function value of -285 . We replace the solutions obtained in this model in objective function of Wang and Vatada's paper and calculated the objective function and the results are shown in Table 18.

Table 18

The objective function value

\begin{tabular}{llllll}
\hline Solution & 1 & 2 & 3 & 4 & 5 \\
\hline OF & -208 & -131 & -143 & -235 & -198 \\
\hline
\end{tabular}

It is noteworthy that all the obtained solutions are slightly worse than solution by article itself, which is normal because none of them is exactly equal to the optimal point. Now, the optimized obtained variables by the article are replaced in the model and the results are summarized in Table 19.

Table 19

Values of objective functions with variables optimized by Wang article

\begin{tabular}{ccc}
\hline First objective function & Second objective function & Third objective function \\
\hline 7.13 & 2250 & 0.21 \\
\hline
\end{tabular}

In this case, with these values, the objective function is worse than the values obtained by the model optimal variables. In summary, we can see that provided model maintains a relatively high efficiency and can be applied according to status quo for selecting the related nodes.

\section{Conclusion}

In this paper, we have proposed a new multi-objective model to locate emergency response centers for transporting the hazardous materials. Since many real-world applications are faced with uncertainty in input parameters, the proposed model of this paper also assumed that reference and demand to such center was subject to uncertainty, where demand was fuzzy random. The resulted problem formulation was modeled as nonlinear non-convex mixed integer programming and we used NSGAII method to solve the resulted problem. The performance of the proposed model has been examined with different examples using various probability distribution and they are compared with the performance of other existing method. 


\section{References}

Berman,O., Krass, D., \& Wang, J. (2011). The probabilistic gradual covering location problem on a network with discrete random demand weights. Computers \& Operations Research, 38(11), 14931500.

Erkut, E., \& Gzara, F. (2008). Solving the hazmat transport network design problem. Computers and Operations Research, 35(7), 2234-2247.

Erkut, E. \& Alp, O. (2007). Designing a road network for hazardous materials shipments. Computers \& Operations Research, 34, 1389-1405.

Kara, B.Y., \& Verter,V. (2004). Designing a road network for hazardous materials transportation. Transportation Science, 38(2), 188-196.

Ke, H., \& Liu, B. (2007). Project scheduling problem with mixed uncertainty of randomness and fuzziness. European Journal of Operational Research, 183(1), 135-147.

Ishii, H. \& Lee, Y.L. \& Yeh, K.Y. (2007) Fuzzy facility location problem with preference of candidate sites. Fuzzy Sets and Systems, 158(17), 1922-1930.

Javanshir, H., Ebrahimnejad, S., \& Nouri, S. (2012). Bi-objective supply chain problem using MOPSO and NSGA-II. International Journal of Industrial Engineering Computations, 3, 681-694.

Liang, R., Gao, K. \& Iwamura, K. (2007). Fuzzy random dependent-chance bilevel programming with applications. Lecture Notes in Computer Science, 4492, 257-266.

Liu, B. (2007). Theory and Practice of Uncertain Programming. UTLAB.

Venkata Rao, R., \& Patel, V. (2012). An elitist teaching-learning-based optimization algorithm for solving complex constrained optimization problems. International Journal of Industrial Engineering Computations, 3(4), 535-560.

Yang, L. Ji, X., Gao, Z., \& Li, K. (2007). Logistics distribution centers location problem and algorithm under fuzzy environment. Journal of Computational and Applied Mathematics, 208(2), 303-315.

Yang,J. \&Xiong,J. \& Liu,S. \& Yang,C.(2008) Flow capturing location-allocation problem with stochastic demand under hurwicz rule. Fourth International Conference on Natural Computation, 7 , 169-173.

Wang, S., \& Watada, J. (2010). A hybrid modified PSO approach to VaR-based facility location problems with variable capacity in fuzzy random uncertainty. Information Sciences, 192(1), 3-18.

Wen, M., \& Iwamura, K. (2008). Facility location-allocation problem in random fuzzy environment: Using $(\alpha, \beta)$-cost minimization model under the Hurewicz criterion. Computers \& Mathematics with Applications, 55(4), 704-713.

Sadjadi, S.J., Pourmoayed, R., \& Aryanezhad, M.B. (2012). A robust critical path in an environment with hybrid uncertainty. Applied Soft Computing, 12(3), 1087-1100.

Schütz,P. \& Stougie,L. \& Tomasgard,A. (2008)Stochastic facility location with general long-run costs and convex short-run costs. Computers \& Operations Research, Vol. 35, No. 9, pp-2988-3000.

US Department of Transportation(2000). Departmentwide program evaluation of the hazardous materials transportation programs. http://hazmat.dot.gov/hmpe_report.pdf 